\title{
Contemporary management of keratoconus... a significant paradigm shift is in place
}

\section{Editorial}

Corneal ectasia can be defined as a thinning disorder associated with progressive corneal steepening and surface irregularity. Forms of corneal ectasia include keratoconus, keratoglobus, pellucid marginal degeneration, and post keratorefractive ectasia. Corneal ectasias are associated with progressive refractive ammetropias (especially irregular astigmatism), reduction of best corrected spectacle visual acuity, increased high order aberrations and visual distortions including monocular diplopia/polyopia, ghost images, halos, etc. Keratoconus is a naturally occurring form of corneal ectasia where the cornea assumes a locally conical shape due to thinning and protrusion. The disease typically has an onset during the late first to second decades of life and will often stabilize beyond the age of 40 . However keratoconus can have quite a variable age of onset and degree of progression. The prevalence of keratoconus is most commonly reported to be about 1 in 2,000 , but with newer technologies allowing for earlier diagnosis and detection of subtle and often "sub-clinical" cases the true prevalence is unknown but thought to be possibly as common as 1 in 500. Genetic factors are being explored that are confirming the hereditary nature of the disease. Keratoconus remains one of the most common reasons for corneal transplantation.

Until relatively recently management of keratoconus involved initial diagnosis, this typically would take place following the report of symptomatology. Diagnosis was based initially on clinical findings found on slit lamp examination, keratometric and retinoscopic measurements and possibly also based on refractive shifts such as atypical increases in astigmatism or significant progression of refractive inter-ocular asymmetry without other apparent causes. More recently with the advent of corneal topography, keratoconuslike topographic anomalies were found independent of other clinical findings. Regardless of where along the continuum of the disease a patient was diagnosed, the only treatments available for keratoconus were contact lens management and if needed some form of keratoplasty when contact lens intolerance was experienced. Early diagnosis had no influence on the ultimate outcome which could range from subclinical to mild to moderate to serve.

Today however technological advances in the early diagnosis of keratoconus and development of treatment strategies that can limit; if not halt progression has resulted in a revolutionary paradigm shift in the contemporary management of the disease. Diagnostic technologies such as corneal tomography, aberrometry, measurement of corneal biomechanics, and high resolution corneal ultrasonography among

\author{
Volume I Issue I - 2014
}

\author{
Steven Barry Eiden \\ North Suburban Vision Consultants, Ltd., USA
}

Correspondence: Steven Barry Eiden, President and Medical Director of North Suburban Vision Consultants, Ltd., Deerfield, Illinois and Park Ridge, Illinois, USA, Tel 8474I203II, Email sbeiden@nsvc.com

Received: August 12, 2014 | Published: August 13, 2014

others are allowing clinicians to detect keratoconus at much earlier and milder stages and in many cases significantly before traditional clinical findings are detected or subjective symptoms experienced. Treatment methods such as corneal collagen cross linking and intracorneal ring segment implantation has been shown to dramatically reduce and in many cases halt the progression of the disease. Combining early detection diagnostic technologies with treatments to control progression can allow us to improve patient's quality of life in dramatic ways and will ultimately reduce the numbers of cases that progress to require corneal transplantation. Additionally, other treatment technologies are being developed that appear to be able to significantly reduce the severity of the disease and improve visual function. It is now imperative to make the earliest diagnosis possible and implement progression control treatment strategies. Further, based on the hereditary nature of keratoconus, it is also imperative to examine blood relatives of our diagnosed keratoconus patients who are likely at risk for having the disease even without experiencing overt symptoms. These are exciting times in the field of keratoconus and corneal ectasia management. Ongoing research will continue to result in amazing new technologies and approaches to the care of patients with these diseases. We as clinicians are obligated to keep up with the most current research and development in this field in order to allow our patients to reap the benefits of this explosion of knowledge and resultant change in our treatment paradigms.

\section{Acknowledgments}

None.

\section{Conflicts of interest}

Author declares that there is no conflict of interest. 